\title{
Direct Pulp Capping With ProRoot MTA Alone and in Combination With Er:YAG Laser Irradiation: A Clinical Trial
}

\author{
Hamid Kermanshah ${ }^{\circledR}$, Ladan Ranjbar Omrani', Sholeh Ghabraei ${ }^{2}$, Reza Fekrazad ${ }^{3,4}$, Niloufar Daneshparvar ${ }^{5}$, \\ Parisa Bagheri ${ }^{* *}$ \\ ${ }^{1}$ Associate Professor, Department of Restorative Dentistry, School of Dentistry, Tehran University of Medical Sciences, \\ Tehran, Iran. \\ ${ }^{2}$ Associate Professor, Department of Endodontics, School of Dentistry, Tehran University of Medical Sciences, Tehran, Iran. \\ ${ }^{3}$ Radiation Science Research Center, Laser Research Center in Medical Sciences, AJA University of Medical Sciences, \\ Tehran, Iran \\ ${ }^{4}$ International Network for Photo Medicine and Photo Dynamic Therapy (INPMPDT), Universal Scientific Education and \\ Research Network (USERN), Tehran, Iran \\ ${ }^{5}$ Postgraduate Student of Periodontics, Indiana University, School of Dentistry. USA \\ ${ }^{6}$ Faculty Member, Department of Endodontics, School of Dentistry, Tehran University of Medical Sciences, Tehran, Iran
}

\section{*Correspondence to Parisa Bagheri DDS, MSc; Faculty Member, Department of Endodontics, School of Dentistry, Tehran University of Medical Sciences, Tehran, Iran. Tel: +989125061348; Email: parisbagheri@gmail.com}

Published online December 30, 2020

\begin{abstract}
Background: Due to the complications of root canal treatment, conservative modalities to preserve pulp vitality are favorable. Direct pulp capping (DPC) refers to the coverage of the pulp tissue exposed by a biocompatible agent that can improve the prognosis of the tooth by $44 \%$ to $95 \%$. Some recent studies have reported more predictable results (a success rate of about 90\%) for DPC with laser and regenerative materials such as mineral trioxide aggregate (MTA). This study aimed to clinically compare DPC with ProRoot MTA alone and in combination with Er:YAG (erbium-doped yttrium aluminium garnet) laser irradiation (2940 nm).

Methods: This clinical trial was conducted on 26 patients with a mean age of 29 years (in the range of 17 to 46 years) who required DPC following pulp exposure during deep caries removal. The teeth were divided into two groups. In the control group, the teeth underwent DPC with ProRoot MTA while in the test group, the teeth were first irradiated with a $2940 \mathrm{~nm}$ Er:YAG laser and then underwent DPC with ProRoot MTA. The patients were recalled at one, 3 and 6 months for the follow-up (clinical and radiographic examinations). The data were analyzed using Fisher exact test. Results: The success rate was $75 \%$ in the laser group and $93 \%$ in the control group. The groups were not significantly different $(P>0.05)$.

Conclusion: No significant difference was found in terms of the success rate of DPC with ProRoot MTA alone and in combination with Er:YAG laser irradiation.

Keywords: Dental pulp capping; Lasers; Solid-state; Mineral trioxide aggregate; ProRoot MTA.
\end{abstract}

\section{Introduction}

Root canal treatment is suitable for teeth with irreversible pulpitis or pulp necrosis. However, considering the functions of dental pulp and its protective mechanisms to confront pathogenic bacteria, pulp vitality should be preserved if possible. ${ }^{1}$

Vital pulp therapy is designed to preserve and maintain pulpal health in teeth that have been exposed to trauma, caries, restorative procedures, and anatomic anomalies. ${ }^{2}$

Vital pulp therapy includes full pulpotomy, partial pulpotomy and direct pulp capping (DPC) and is a biological, affordable, conservative and simple modality with a high success rate. It is recommended for all teeth diagnosed with reversible pulpitis or partially inflamed pulps in which the remaining healthy tissue can be conserved to generate a hard tissue barrier that seals and protects the pulp from a future microbial insult. ${ }^{3-5}$

The underlying purpose of vital pulp therapy is to avoid or delay root canal therapy and advanced restorative care because these, together, may reduce long-term tooth survival compared to teeth with vital pulp. ${ }^{6}$

$D P C$ is defined as "placing a dental material directly on mechanical or traumatic vital pulp exposure" and "sealing the pulpal wound to facilitate the formation of reparative dentin and the maintenance of the vital pulp."

DPC is a common treatment modality in restorative 
dentistry and endodontics because successful pulp capping can maintain the vitality of the exposed pulp and consequently the tooth, which is important from both the biomechanical and esthetic points of view. ${ }^{5}$

The success of DPC depends on case selection, hemostatic agents, the choice of pulp capping material, the integrity of the sealed permanent restoration, the time interval between pulp exposure and treatment, patient's age, the developmental stage of the root, the location of pulp exposure, and the severity of bleeding. Its success rate may range from $44 \%$ to $95 \%{ }^{5,6,8-11}$ In cases of traumatic pulp exposure, early intervention during the first 24 hours provides the best prognosis since it minimizes microbial accumulation. ${ }^{8}$

The introduction of new bioactive materials, along with modified protocols, makes more teeth with deep caries, traumatic injuries, and mechanical exposure viable candidates for innovative pulp therapies designed to potentiate and maintain pulpal survival. ${ }^{6,9-11}$

At present, several methods are available for DPC. Mineral trioxide aggregate (MTA) has suitable properties such as high sealing ability, ${ }^{12}$ high $\mathrm{pH}^{12}$ optimal biocompatibility, prevention of bacterial leakage, ${ }^{13}$ and long-term stability.

MTA stimulates reparative hard tissue formation by sequestering growth factors and cytokines and other bioactive molecules embedded in the surrounding dentin matrix, such as transforming growth factor beta (TGF- $\beta$ ), adrenomedullin, vascular endothelial growth factor, macrophage colony-stimulating factor, interleukins (IL$1 \alpha$, IL-1 $\beta$, Il-6, and IL-8), myeloperoxidase, inducible nitric oxide synthase, nuclear factor-kappa $B$, activating protein- 1 , and cyclooxygenase- $2 .^{14-18}$

MTA most likely up-regulates the fibroblast secretion of BMP-2 and TGF- $\beta 1 .^{18-23}$ The increased presence of these dentine extracellular proteins as the result of MTA culminates in dentin bridge formation after stimulating the reparative dentinogenic mechanism. ${ }^{14,24,25}$

Lasers are among the currently used methods for pulp capping. Recent studies have reported a success rate of about $90 \%$ for DPC with a laser. ${ }^{26}$ Lasers for pulp capping have advantages such as disinfecting and coagulative properties, ${ }^{8}$ bactericidal effects, and providing a sterile environment. It should be noted that all laser types have sterilizing effects. Considering the key role of elimination of bacteria in the success of DPC, the sterilizing effect of a laser may be able to enhance the success rate of this treatment modality. ${ }^{8}$

Studies on the effect of the Er:YAG (erbium-doped yttrium aluminium garnet) laser on dental pulp have discussed that Er:YAG laser irradiation triggers reparative pulp response, the formation of the dentinal bridge, and the prevention of secondary caries..$^{27}$ This study aimed to assess the effect of the Er:YAG laser on the success rate of DPC with MTA.

\section{Materials and Methods}

This clinical trial was conducted on patients referred to the School of Dentistry, Tehran University of Medical Sciences. The inclusion criteria were as follows:

- Deep caries with less than $1 \mathrm{~mm}$ distance to dental pulp on periapical radiographs

- Absence of spontaneous/nocturnal pain, fistula, periapical lesion or any sign/symptom of irreversible pulpitis

- Permanent teeth with closed apices

- No mobility

- No sensitivity to percussion

- No periodontal problem

- The tooth was not supposed to be an abutment for denture or orthodontic appliances

- Patients did not have bruxism

- Patients were not under chemotherapy or radiotherapy

After taking a periapical radiograph and clinical photographs and conduction of clinical heat (with a burnisher heated on flame) and cold (cotton pellet and ethylene chloride spray) tests, patients between 17 and 46 years were chosen for the study. They were briefed about the treatment and its risk of failure and signed informed consent forms.

A rubber dam (Dental Dam, USA) was placed and local anesthesia was induced by injection. Cavity outline was prepared by a diamond bur, and then carious lesions were removed by a carbide bur. First, the peripheral caries was removed and then the pulpal wall caries was carefully removed using a low-speed hand-piece. Of 56 teeth in the study, pulp exposure occurred in 26 during caries removal.

The remaining teeth were restored with amalgam or composite resin. In the case of pulp exposure, the size of exposure was measured by a periodontal probe and then bleeding was controlled using a cotton pellet dipped in $2 \%$ sodium hypochlorite. The diagnosis of irreversible pulpitis was made if the bleeding did not stop within 10 minutes and such teeth were excluded from the study.

After hemostasis, the patient was assigned to the laser group or the control group. In the laser group, first the exposure area and the entire cavity floor were irradiated with the Er:YAG laser with a $2940 \mathrm{~nm}$ wavelength (wavelength $100 \mathrm{~mJ}$ energy per pulse, $10 \mathrm{~Hz}$ repetition rate, 450 micro-second pulse duration and $1 \mathrm{~mm}$ tip diameter) with minimal distance from the exposure site was used for 10 seconds in a scanning mode on the site with $0.5 \mathrm{~mm}$ of the surrounding tissues.)

In order to prevent the tissue from peeling, air and water parameters were turned off) (Figure 1). Then ProRoot MTA (Dentsply) was applied to the exposed area for pulp capping.

MTA powder was mixed with distilled water with a 3:1 ratio on a glass slab to reach a creamy consistency and it was then applied over the exposure site using an 
MTA carrier. A cotton pellet dipped in distilled water was placed on the MTA and then the cavity was temporarily restored with polycarboxylate cement (P25, Germany).

The same procedures were performed in the control group except for laser irradiation.

The patients were recalled 1 week later. The temporary restoration was removed and the MTA was assessed in terms of hardness, coverage of the exposure site and sealing of the area. The temporary restoration was removed and the MTA setting was assessed. MTA had been displaced in three patients ( 1 from the control group and 2 from the laser group), so in these three patients MTA was placed on the exposure site again.

After ensuring an adequate MTA setting, a wedge (Minadent, Iran) and a metal matrix band were placed and the enamel edges were etched (Meta Etchant, Korea) for 15-30 seconds, washed and dried. Then two layers of a $6^{\text {th }}$ generation bonding agent (Clearfil SE Bond, Okayama, Japan) were applied over the cavity of all of the teeth of both groups and they were cured with a light-curing unit (Woodpecker, China) with a light intensity of $1000 \mathrm{~mW} /$ $\mathrm{cm}^{2}$ for 20 seconds. The cavity was incrementally filled with composite resin (Z250, 3M ESPE, USA) and cured with a light-curing unit (Woodpecker, China) with a light intensity of $1000 \mathrm{~mW} / \mathrm{cm}^{2}$ for 40 seconds, or it was filled with high-copper amalgam (Sinalux, Iran) (Figure 2).

Cavities were class II, class V or complex. Of all teeth, seven were restored with amalgam, 19 were restored with composite and four teeth received complex restorations (Choosing different filling materials was based on the extent and shape of the cavity (subgingival position of cavosurface margin), esthetic (composite vs. amalgam), and most importantly establishing a coronal seal using either material).

The patients were followed up for one, 3 and 6 months. Cold and heat tests were performed and radiographs were obtained on the follow-up sessions. In case of noticing signs/symptoms of tooth necrosis during the follow-up period, the patient was referred for endodontic treatment (4 patients; 3 from the laser group and 1 from the control

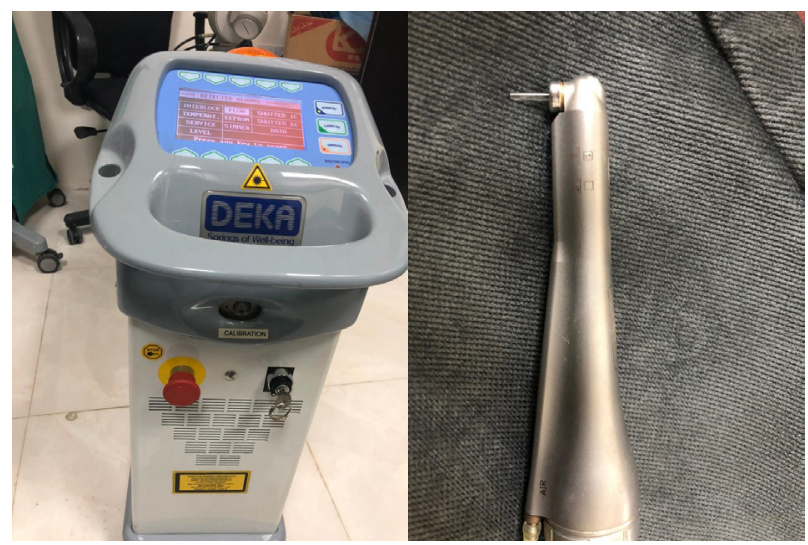

Figure 1. The Laser Device and Handpiece.

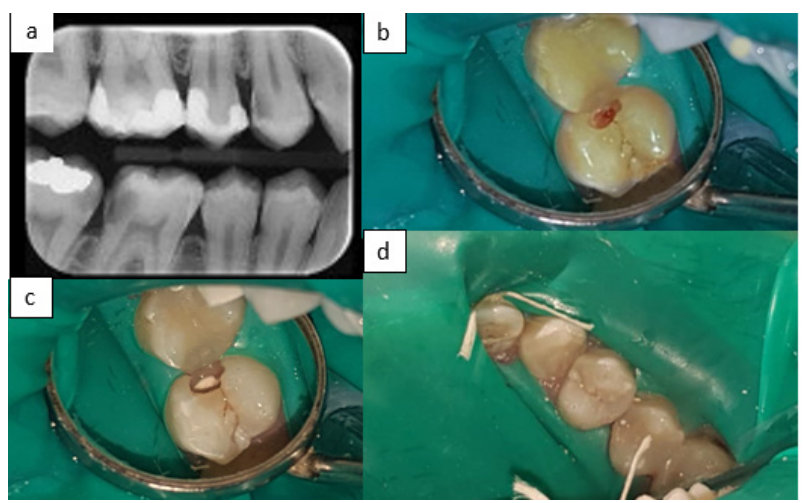

Figure 2. (a) Pre-operative Radiograph, (b) Pulp Exposure During Caries Removal, (c) Applying ProRoot MTA (Dentsply) Over the Exposed Area After Hemostasis (Using a Cotton Pellet Dipped in 2\% Sodium Hypochlorite), (d) Permanent Restoration With Composite Resin (z250,3M ESPE, USA).

group).

Fisher exact test was applied to compare the percentage of success between the two groups with a $P<0.05$ level of significance.

\section{Results}

The control group included 14 patients between 17 to 46 years old (the mean patient age was 29). The laser group included 12 patients between 18 to 46 years old (the mean patient age was 28). Table 1 shows patient demographics and type of teeth.

The control group (14 teeth) included 11 small, 1 medium and 2 large exposures. The laser group (12 teeth) included 2 small, 3 medium and 7 large exposures. In the control group, 13 out of 14 teeth (93\%) remained vital during the 3-month follow-up. One tooth that had small exposure did not respond to pulp sensibility tests at a onemonth follow-up and underwent RCT.

9 out of 12 teeth (75\%) in the laser group remained vital during the 3-month follow-up. All three teeth that did not respond to pulp sensibility tests at follow-up sessions and underwent RCT had large exposures. One tooth in the laser group showed treatment success at the one-month follow-up but failed two months later. Other failures occurred during the first month postoperatively.

The percentage of success was not significantly different between the two groups $(P$ value $=0.31)$.

Table 2 shows the percentage of the vitality of the teeth in the laser and control groups at one and three months. 7 out of 13 patients $(53.8 \%)$ in the control group and 1 out of 9 patients $(11.11 \%)$ in the laser group showed up for a six-month follow-up, in which the teeth responded to cold and heat tests. The other 9 patients had no complaint of symptoms and did not show up. Radiographic evaluation at 1, 3 and 6 months showed no signs of periapical disease.

\section{Discussion}

Evidence shows that conservative treatments to preserve 
Table 1. Patient Demographics and Type of Teeth That Received Pulp Capping Treatment

\begin{tabular}{llcc}
\hline & & Control & Laser \\
\hline \multirow{2}{*}{ Gender } & Male & 5 & 7 \\
\multirow{2}{*}{ Age } & Female & 9 & 5 \\
& & $29 \pm 9$ & $28 \pm 10$ \\
\cline { 2 - 2 } Type of tooth & Canine & 1 & 0 \\
& First premolar & 2 & 3 \\
& First molar & 3 & 5 \\
& Second molar & 5 & 4 \\
\hline
\end{tabular}

Table 2. Percentage of the Vitality of Teeth in the Laser and Control Groups at 1 and 3 Months

\begin{tabular}{lcc}
\hline & 1 Month & 3 Months \\
\hline Control & $93 \%$ & $93 \%$ \\
Laser & $83 \%$ & $75 \%$ \\
\hline
\end{tabular}

pulp vitality are increasing. ${ }^{28}$ DPC is defined as the coverage of dental pulp with a biocompatible material such as MTA following exposure due to caries or trauma. This treatment modality can acceptably improve the prognosis of such teeth. ${ }^{29}$

In the current study, DPC was performed for 26 teeth with extensive caries, which experienced pulp exposure following caries removal. The teeth were subjected to DPC with ProRoot MTA alone or in combination with Er:YAG laser irradiation. Recent studies have reported more predictable results (about 90\% success rate) for DPC with lasers compared to conventional techniques (about $60 \%$ success rate). Studies on the application of lasers with different wavelengths for vital pulp therapy are limited. ${ }^{8}$ DPC with lasers has significant advantages compared to conventional DPC including:

1. Disinfecting effect which is due to the bactericidal properties of laser irradiation. All laser types possess this property; however, the disinfecting efficacy of a laser may vary depending on its wavelength and penetration depth. $\mathrm{CO}_{2}$ and erbium lasers have a more superficial effect than diode and Nd:YAG lasers.

2. Coagulation: Lasers cause superficial necrosis of the area with reversible pulpitis and eventually lead to dentinal bridge formation.

3. Insignificant temperature rise: Erbium lasers with 2780 to $2940 \mathrm{~nm}$ wavelengths do not significantly increase the temperature since they are used in a noncontact mode and remove the tissue under copious water and air spray.

4. Decompression of cavity: Erbium lasers cause decompression of the cavity and decrease the risk of entry of infected dentinal chips into the pulp.

5. Dentinal melting: Erbium lasers, particularly the Er,Cr:YSGG laser, create a barrier against physical and chemical stimuli.

6. Biostimulating effect: Erbium lasers have high potential for pulpal repair and regeneration., ${ }^{5,826,27}$

The Er:YAG laser enables the coagulation and disinfection of exposed pulp. The coagulating effect of this type of laser is controllable, in contrast to other wavelengths of the laser, due to its superficial penetration. . $^{5,26,27}$

Considering the advantages of lasers for DPC, especially erbium lasers, as well as the limited studies available on this topic, we evaluated the effect of Er:YAG laser irradiation on the outcome of DPC with MTA. Since cavity preparation was not performed with a laser in this study, we did not expect decompression or an insignificant increase in pulpal temperature. We used a laser for the purpose of coagulation and disinfection of the exposure site. Moreover, we used ProRoot MTA for DPC in both laser and control groups since it has optimal properties such as high sealing ability, high $\mathrm{pH}$, optimal biocompatibility, prevention of bacterial leakage, longterm stability and induction of cementum, and bone and dentin formation ${ }^{12,13,16,24,29}$ and it has an optimal success rate for DPC.

In an animal study in 2001, Jayawardena et al histologically evaluated the pulpal response to Er:YAG laser irradiation following accidental pulpal exposure and concluded that this laser was highly capable of the induction of the dentinal bridge and secondary dentin formation. ${ }^{27}$ Our results were in agreement with those of Olivi et al, ${ }^{8}$ who reported a $75 \%$ success rate in the Er:YAG laser group. However, they prepared the cavity with a laser while we prepared the cavity using a bur. Cavity preparation by a laser provides better conditions for pulpal repair. On the other hand, patients were younger in their study compared to ours.

Santucci et al in 1997 were the first to use the Nd:YAG laser for DPC in humans. They compared DPC with calcium hydroxide and laser in terms of the success rate and showed that the laser group had a higher success rate. Their results were different from ours, which may be due to the use of calcium hydroxide instead of MTA. Also, They used the Nd:YAG laser instead of the Er:YAG laser. ${ }^{26}$

In 2013, Hilton et al ${ }^{30}$ compared the success rate of DPC with MTA and calcium hydroxide and reported that the success rate of MTA at the two-year follow-up was $81 \%$ while this rate was $78.5 \%$ for calcium hydroxide. The success rate of DPC with MTA was $93 \%$ in our study. This small difference may be attributed to different follow-up periods. ${ }^{30}$

In a review study by Aguilar and Linsuwanont in 2011, the mean success rate of MTA at one year was $90.5 \%$, which was in agreement with our findings. ${ }^{31}$

Hasheminia et $\mathrm{al}^{29}$ histologically compared DPC with the Er:YAG laser and MTA in their animal study and showed that DPC with MTA alone and in combination with Er:YAG laser irradiation can both yield optimal 
results. Their findings were in agreement with ours.

In 1995, Moritz et $\mathrm{al}^{5}$ evaluated the results of DPC with the $\mathrm{CO} 2$ laser and reported the two-year success rate of $93 \%$ in the laser group and $68 \%$ in the calcium hydroxide (control) group. Their findings were different from ours, which may be explained by the use of different types of lasers, different follow-up times and different types of pulp exposure (they evaluated cases of accidental pulp exposure with no carious lesion).

In the case of pulp exposure following extensive caries, it would be difficult to accurately evaluate the pulp status. Although the assessment of pulp status plays a critical role in the success of vital pulp therapy, there is no accurate tool to determine the degree of inflammation of dental pulp. Clinical symptoms such as the presence and severity of pain and cold, heat and electrical pulp tests only show a positive or negative response. ${ }^{32}$ Histological studies have shown that exposed pulp tissue following carious pulp exposure is not always infected; this depends on the time passed since exposure and severity of caries. ${ }^{31}$ Matsuo et al believed that the severity of bleeding following pulp exposure is a better indicator of the severity of pulpal inflammation compared to clinical symptoms. Severe bleeding, which is difficult to control, usually indicates severe pulpal inflammation. ${ }^{32}$ Several methods have been recommended to control pulpal bleeding. Irrigation with sodium hypochlorite (1.25\% to $6 \%$ ) is the most commonly suggested technique. If bleeding does not stop within 1-10 minutes, it may show incomplete removal of infected pulp tissue or the spread of infection into the radicular pulp. ${ }^{31-}$ 38

Thus, in the current study, clinical examination was carried out by the heat and cold tests to rule out irreversible pulpitis. In the case of pulp exposure, first the area was rinsed with chlorhexidine and we tried to stop the bleeding by placing a cotton pellet dipped in sodium hypochlorite over the exposure site. If the bleeding did not stop within 10 minutes, the diagnosis of irreversible pulpitis was made and the patient was excluded from the study.

Studies on the effect of age on the success of vital pulp therapy are scarce. This treatment modality is more commonly recommended for young adults because it is believed that young individuals' pulp tissue has high regeneration potential. However, no clinical study is available to confirm this statement and patients in the age range of 6 to 70 years have well responded to vital pulp therapy. This indicates the high potential of dental pulp for regeneration following the elimination of infective agents. $^{31}$

In the current study, the patients were between 17 to 46 years of age (mean age of 29 years). Teeth with failed pulp capping treatment belonged to those who were 45, 35, 33 and 46 years old.

Considering the results of previous studies on the insignificant effect of the size of exposure on the results of DPC, ${ }^{39}$ this factor was not evaluated in our study. Due to the existing limitations, randomization was not performed. The majority of teeth in the laser group (7 out of 12 teeth) had large (>2 mm) exposure sites while the majority of teeth in the control group (10 out of 14 teeth) had small exposure sites $(<1 \mathrm{~mm})$. Also, all failures in the laser group occurred in the teeth with large exposure sites. Therefore, it may be assumed that the large size of exposure may be responsible for a lower success rate in this group.

On the other hand, the follow-up period was short in our study (6 months). Considering the reported success rates for DPC with a laser in long-term follow-ups (over 2 years), it may be expected that the successful treatments last for a long time in the laser group.

According to the current results, DPC with MTA had a 93\% success rate while DPC with MTA and the Er:YAG laser showed a $75 \%$ success rate. Therefore, both modalities are acceptable for the preservation of pulp vitality, although it seems that DPC with MTA and the Er:YAG laser with the parameters used in this study has no superiority over DPC with MTA alone.

Since the difference in the success rate between the two groups was not significant with the selected sample size, this study can serve as a pilot study for future studies on this topic.

\section{Ethical Considerations}

The study was approved by the ethics committee of Tehran University of Medical Sciences (Ethics code: -04-97-19119-8171191) Additionally, this study was registered in the Iranian Registry of Clinical Trials (identifier: IRCT2013102015078N1; https://www.irct. ir/trial/14469). Informed consent was obtained from all individual participants included in the study.

\section{Conflict of Interests}

The authors declare that they have no conflict of interest.

\section{Acknowledgements}

The authors wish to thank Dr. AR. Shamshiri for his assistance in doing statistical analysis.

\section{Funding}

This study was funded by Tehran University of Medical Sciences.

\section{References}

1. Ward J. Vital pulp therapy in cariously exposed permanent teeth and its limitations. Aust Endod J. 2002;28(1):29-37. doi: 10.1111/j.1747-4477.2002.tb00364.x.

2. Cho SY, Seo DG, Lee SJ, Lee J, Lee SJ, Jung IY. Prognostic factors for clinical outcomes according to time after direct pulp capping. J Endod. 2013;39(3):327-31. doi: 10.1016/j. joen.2012.11.034.

3. Al-Hiyasat AS, Barrieshi-Nusair KM, Al-Omari MA. The 
radiographic outcomes of direct pulp-capping procedures performed by dental students: a retrospective study. $J \mathrm{Am}$ Dent Assoc. 2006;137(12):1699-705. doi: 10.14219/jada. archive.2006.0116.

4. Swift Jr EJ, Trope M, Ritter AV. Vital pulp therapy for the mature tooth - can it work? Endod Topics. 2003;5(1):49-56. doi: 10.1111/j.1601-1546.2003.00030.x.

5. Moritz A, Schoop U, Goharkhay K, Sperr W. The $\mathrm{CO}_{2}$ laser as an aid in direct pulp capping. J Endod. 1998;24(4):24851. doi: 10.1016/S0099-2399(98)80106-4.

6. Mjör IA. Pulp-dentin biology in restorative dentistry. Part 5: Clinical management and tissue changes associated with wear and trauma. Quintessence Int. 2001;32(10):771-88.

7. American Association of Endodontists. Glossary of endodontic terms. Chicago: American Association of Endodontists; 2012.

8. Olivi G, Genovese MD, Maturo P, Docimo R. Pulp capping: advantages of using laser technology. Eur J Paediatr Dent. 2007;8(2):89-95.

9. Caplan DJ, Cai J, Yin G, White BA. Root canal filled versus non-root canal filled teeth: a retrospective comparison of survival times. J Public Health Dent. 2005;65(2):90-6. doi: 10.1111/j.1752-7325.2005.tb02792.x.

10. Caplan DJ, Kolker J, Rivera EM, Walton RE. Relationship between number of proximal contacts and survival of root canal treated teeth. Int Endod J. 2002;35(2):193-9. doi: 10.1046/j.1365-2591.2002.00472.x.

11. Ng YL, Mann V, Gulabivala K. A prospective study of the factors affecting outcomes of non-surgical root canal treatment: part 2: tooth survival. Int Endod J. 2011;44(7):610-25. doi: 10.1111/j.1365-2591.2011.01873.x.

12. Ford TR, Torabinejad M, Abedi HR, Bakland LK, Kariyawasam SP. Using mineral trioxide aggregate as a pulpcapping material. J Am Dent Assoc. 1996;127(10):1491-4. doi: 10.14219/jada.archive.1996.0058.

13. Abedi HR, Ingle JI. Mineral trioxide aggregate: a review of a new cement. J Calif Dent Assoc. 1995;23(12):36-9.

14. Koh ET, McDonald F, Pitt Ford TR, Torabinejad M. Cellular response to mineral trioxide aggregate. J Endod. 1998;24(8):543-7. doi: 10.1016/S0099-2399(98)80074-5.

15. Koh ET, Torabinejad M, Pitt Ford TR, Brady K, McDonald F. Mineral trioxide aggregate stimulates a biological response in human osteoblasts. J Biomed Mater Res. 1997;37(3):4329. doi: 10.1002/(sici)1097-4636(19971205)37:3<432::aidjbm14>3.0.co;2-d.

16. Tziafas D, Pantelidou O, Alvanou A, Belibasakis G, Papadimitriou S. The dentinogenic effect of mineral trioxide aggregate (MTA) in short-term capping experiments. Int Endod J. 2002;35(3):245-54. doi: 10.1046/j.13652591.2002.00471.x.

17. Matsumoto S, Hayashi M, Suzuki Y, Suzuki N, Maeno $\mathrm{M}$, Ogiso B. Calcium ions released from mineral trioxide aggregate convert the differentiation pathway of $\mathrm{C} 2 \mathrm{C} 12$ cells into osteoblast lineage. J Endod. 2013;39(1):68-75. doi: 10.1016/j.joen.2012.10.006.

18. Paranjpe A, Zhang H, Johnson JD. Effects of mineral trioxide aggregate on human dental pulp cells after pulpcapping procedures. J Endod. 2010;36(6):1042-7. doi: 10.1016/j.joen.2010.02.013.

19. D’Antò V, Di Caprio MP, Ametrano G, Simeone M, Rengo S, Spagnuolo G. Effect of mineral trioxide aggregate on mesenchymal stem cells. J Endod. 2010;36(11):1839-43. doi: 10.1016/j.joen.2010.08.010.

20. Guven G, Cehreli ZC, Ural A, Serdar MA, Basak F. Effect of mineral trioxide aggregate cements on transforming growth factor betal and bone morphogenetic protein production by human fibroblasts in vitro. J Endod. 2007;33(4):447-50. doi: 10.1016/j.joen.2006.12.020.

21. Seo MS, Hwang KG, Lee J, Kim H, Baek SH. The effect of mineral trioxide aggregate on odontogenic differentiation in dental pulp stem cells. J Endod. 2013;39(2):242-8. doi: 10.1016/j.joen.2012.11.004.

22. Takita T, Hayashi M, Takeichi O, Ogiso B, Suzuki N, Otsuka $\mathrm{K}$, et al. Effect of mineral trioxide aggregate on proliferation of cultured human dental pulp cells. Int Endod J. 2006;39(5):415-22. doi: 10.1111/j.1365-2591.2006.01097.x.

23. Yasuda Y, Ogawa M, Arakawa T, Kadowaki T, Saito T. The effect of mineral trioxide aggregate on the mineralization ability of rat dental pulp cells: an in vitro study. J Endod. 2008;34(9):1057-60. doi: 10.1016/j.joen.2008.06.007.

24. Schönenberger Göhring K, Lehnert B, Zehnder M. [Uses of MTA, a review. Part 2: Clinical applications]. Schweiz Monatsschr Zahnmed. 2004;114(3):222-34. [German, French].

25. Andelin WE, Shabahang S, Wright K, Torabinejad M. Identification of hard tissue after experimental pulp capping using dentin sialoprotein (DSP) as a marker. J Endod. 2003;29(10):646-50. doi: 10.1097/00004770200310000-00008.

26. Santucci PJ. Dycal versus Nd:YAG laser and Vitrebond for direct pulp capping in permanent teeth. J Clin Laser Med Surg. 1999;17(2):69-75. doi: 10.1089/clm.1999.17.69.

27. Jayawardena JA, Kato J, Moriya K, Takagi Y. Pulpal response to exposure with Er:YAG laser. Oral Surg Oral Med Oral Pathol Oral Radiol Endod. 2001;91(2):222-9. doi: 10.1067/ moe.2001.111943.

28. Assif D, Gorfil C. Biomechanical considerations in restoring endodontically treated teeth. J Prosthet Dent. 1994;71(6):565-7. doi: 10.1016/0022-3913(94)90438-3.

29. Hasheminia SM, Feizi G, Razavi SM, Feizianfard M, Gutknecht N, Mir M. A comparative study of three treatment methods of direct pulp capping in canine teeth of cats: a histologic evaluation. Lasers Med Sci. 2010;25(1):915. doi: 10.1007/s10103-008-0584-9.

30. Hilton TJ, Ferracane JL, Mancl L; Northwest Practicebased Research Collaborative in Evidence-based Dentistry. Comparison of $\mathrm{CaOH}$ with MTA for direct pulp capping: a PBRN randomized clinical trial. J Dent Res. 2013;92(7 Suppl):16S-22S. doi: 10.1177/0022034513484336.

31. Aguilar P, Linsuwanont P. Vital pulp therapy in vital permanent teeth with cariously exposed pulp: a systematic review. J Endod. 2011;37(5):581-7. doi: 10.1016/j. joen.2010.12.004.

32. Matsuo T, Nakanishi T, Shimizu H, Ebisu S. A clinical study of direct pulp capping applied to carious-exposed pulps. J Endod. 1996;22(10):551-6. doi: 10.1016/S00992399(96)80017-3.

33. Witherspoon DE, Small JC, Harris GZ. Mineral trioxide aggregate pulpotomies: a case series outcomes assessment. J Am Dent Assoc. 2006;137(5):610-8. doi: 10.14219/jada. archive.2006.0256.

34. Barthel CR, Rosenkranz B, Leuenberg A, Roulet JF. Pulp 
capping of carious exposures: treatment outcome after 5 and 10 years: a retrospective study. J Endod. 2000;26(9):5258. doi: 10.1097/00004770-200009000-00010.

35. DeRosa TA. A retrospective evaluation of pulpotomy as an alternative to extraction. Gen Dent. 2006;54(1):37-40.

36. Bogen G, Kim JS, Bakland LK. Direct pulp capping with mineral trioxide aggregate: an observational study. $J$ Am Dent Assoc. 2008;139(3):305-15. doi: 10.14219/jada. archive.2008.0160

37. Mass E, Zilberman U. Clinical and radiographic evaluation of partial pulpotomy in carious exposure of permanent molars. Pediatr Dent. 1993;15(4):257-9.

38. Mente J, Geletneky B, Ohle M, Koch MJ, Friedrich Ding PG, Wolff D, et al. Mineral trioxide aggregate or calcium hydroxide direct pulp capping: an analysis of the clinical treatment outcome. J Endod. 2010;36(5):806-13. doi: 10.1016/j.joen.2010.02.024.

39. Stanley HR. Criteria for standardizing and increasing credibility of direct pulp capping studies. Am J Dent. 1998;11(Spec No):S17-34. 